\title{
Teleportation: Dream or Reality?
}

\author{
Lev Vaidman \\ March 28, 2021 \\ School of Physics and Astronomy \\ Raymond and Beverly Sackler Faculty of Exact Sciences \\ Tel Aviv University, Tel-Aviv 69978, Israel.
}

\begin{abstract}
Since its discovery in 1993, we witness an intensive theoretical and experimental effort centered on teleportation. Very recently it was claimed in the press that "quantum teleportation has been achieved in the laboratory" (T. Sudbery, Nature 390, 551). Here, I briefly review this research focusing on the connection to nonlocal measurements, and question Sudbery's statement. A philosophical inquiry about the paradoxical meaning of teleportation in the framework of the many-worlds interpretation is added.
\end{abstract}

\section{The meaning of the Word "Teleportation"}

Let me start with a citation from the Oxford English Dictionary [1]:

teleportation. Psychics and Science Fiction. The conveyance of persons (esp. of oneself) or things by psychic power; also in futuristic description, apparently instantaneous transportation of persons, etc., across space by advanced technological means.

Recently, the word "teleportation" has appeared outside of the realm of mystical and science fiction literature: in science journals. Bennett, Brassard, Crepeau, Jozsa, Peres, and Wootters (BBCJPW) [6] proposed a gedanken experiment they termed "quantum teleportation".

Classically, to move a person is to move all the particles it is made of. However, in quantum theory particles themselves do not represent a person: all objects are made of the same elementary particles. An electron in my body is identical to an electron in the paper of the page you are reading now. An object is characterized by the quantum state of the particles it is made of. Thus, reconstructing the quantum state of these particles on other particles of the same kind at a remote location is "transportation" of the object. 
The quantum state of the object to be transported is supposed to be unknown. Indeed, usually we do not know and cannot find out what the quantum state of an object is. Moreover, frequently an object is not in a pure quantum state, its particles may be correlated to other systems. In such cases the essence of the object is these correlations. In order to transport such correlations (even if they are known), without access to the systems which are in correlation with our system, a method for teleportation of an unknown quantum state is necessary.

Quantum teleportation [6] transfers the quantum state of a system and its correlations to another system. Moreover, the procedure corresponds to the modern meaning of teleportation: an object is disintegrated in one place and a perfect replica appears at another site. The object or its complete description is never located between the two sites during the transportation. Note that "disintegration" of the quantum state is a necessary requirement due to the no-cloning theorem.

The teleportation procedure, apart from preparing in advance the quantum channels, requires telegraphing surprisingly small amounts of information between the two sites. This stage prevents "instantaneous" transportation. Indeed, because of special relativity, we cannot hope to achieve superluminal teleportation: objects carry signals.

Due to the arguments presented above, I find the BBCJPW procedure to be very close to the concept of "teleportation" as it is used in the science-fiction literature. However, the name teleportation is less justified for the recent implementations of this idea in the laboratory, as well as for some other proposals for experiments. For me, an experiment deserves the name "teleportation" if I can give to Alice (the sender) a system whose quantum state in unknown to her and that she can, without moving this system and without moving any other system which can carry the quantum state of the system, transport this state to Bob (the receiver) which is located at a remote location. In the next section I shall discuss, in the light of my definition, the usage of the word "teleportation".

\section{Recent Experiments and Proposals for Experiments Termed "Teleportation"}

What I discuss in this section is essentially a semantic issue, but I feel that its clarification is important. I find the original teleportation paper to be one of the most important results in the field in the last ten years, and I think that it should be clearly distinguished from other interesting but less profound achievements.

Recently I heard the word "teleportation" in the context of NMR-type quantum computation experiments [3]. Using certain pulses, a spin state of a nucleus in a large molecule is transported to another nucleus in the same molecule. The main deficiency of this experiment as teleportation is that it does not allow to transport an unknown quantum state. Indeed, in the NMR experiments a macroscopic number of molecules have to be in a particular quantum state. If Alice receives a single quantum object in an unknown 
quantum state, she cannot duplicate it and in that manner prepare many copies in many molecules, due to the no-cloning theorem.

An apparent weakness of the NMR experiment is that the internal coupling which plays the role of the channel for classical information required for teleportation can, in principle, carry the quantum state. However, due to the strong interaction with the environment, the quantum state transmitted through such a channel is effectively measured by the environment. Only the eigenstates corresponding to the classical outcomes are stable under this interaction and, therefore, there is good reason to consider this channel to be classical.

Another place in which I encountered the word "teleportation" is the work on optical simulation of quantum computation [4. It includes a proposal for implementation of the idea to view "teleportation" as a particular quantum computation circuit [5, 6]. The problem in the optical experiment is that instead of the classical channel which is supposed to transmit two bits of information, real photons are moving from Alice to Bob and these photons can transmit the whole quantum state of the polarization degree of freedom of the photon. This is exactly the apparent weakness of the NMR-teleportation experiments mentioned above, but in the present case the environment does not make the quantum channel to be effectively classical. Note that in the original proposal the quantum channel is explicitly replaced by a classical one to make the proposal akin to teleportation in the BBCJPW sense. It is the optical simulation of this proposal which is something less than teleportation. It seems that the authors [4] were aware of this problem when they added a footnote: "The term teleportation is used in the literature to refer to the transfer of the state of a qubit to another". I find this meaning to be too general. Many processes corresponding to this definition were proposed (and even implemented in laboratories) long before the teleportation paper has appeared.

Next, let me discuss "teleportation" in the Rome experiment [7]. As I will explain in the next section, the main obstacle for successful reliable teleportation is the experimental difficulty to make one quantum object interact with another. In optical experiments quantum objects, photons, interact with classical objects such as beam splitters, detectors, etc. Popescu [8] proposed a very elegant solution: two degrees of freedom of a single photon do interact effectively one with the other. This idea was successfully implemented in the Rome experiment in which the polarization state of the photon was transported to another photon. However, the weakness of this experiment is that the quantum state to be teleported has to be the state of (the second degree of freedom of) one of the members of the EPR pair which constitute the quantum channel of the teleportation experiment. Therefore, this method cannot be used for teleportation of an unknown quantum state of an external system. The authors [7] view this experiment as "teleportation" because after the preparation Alice cannot find out the quantum state, which, nevertheless, is transported (always and with high fidelity) to Bob.

Finally, let me discuss the Innsbruck teleportation experiment [9, 10]. Although the word "teleportation" appears in the title of the first Letter [9], the second experiment [10] 
is a much better demonstration of teleportation. I believe that the Innsbruck experiment deserves the name teleportation. It showed for the first time that an unknown state of an external photon can be teleported. It is not a reliable teleportation: the experiment has a theoretical success rate of $25 \%$ only, and the employed methods cannot, in principle, lead to reliable teleportation. For a system consisting of $N$ qubits the probability of successful teleportation is exponentially small.

Recently, Braunstein and Kimble [11] pointed out a weak point of the Innsbruck experiment. In the current version of the experiment one might know that teleportation has been successful only after the time Bob detects (and, therefore, destructs) the photon with the teleported state. Thus, the name given by Braunstein and Kimble for the Innsbruck experiment: "a posteriori teleportation" appears to be appropriate. However, as mentioned in the reply [12 and in the comment itself, it is feasible to solve this problem by a modification of the experiment and therefore it is not a conceptual difficulty.

Another possible improvement of the demonstration of teleportation in the Innsbruck experiment is using single input photons. In the current version of the experiment, the polarizer which controls the input quantum state is stationary, and, therefore, many photons are created in the same state. Thus, this state can hardly be considered an "unknown" quantum state. Low intensity of the input beam and frequent changing of the angle of the polarizer is a simple and effective solution of the problem. An ideal solution is using a "single-photon gun" [13] which creates single-photon states.

Apart from the impossibility of performing a measurement of the nondegenerate Bell operator, there is another problem for achieving reliable teleportation of an unknown state of a single photon. Today, there is no source which creates a single EPR pair at will, something frequently called an "event-ready" source. The second Innsbruck experiment [10] is the best achievement in this direction: entanglement swapping may be viewed as creation of an entangled pair at the moment of the coincidence detection of the two photons coming from the beam splitter. What is missing is a "sophisticated detection procedure" [10] which rules out the creation of two pairs in a single crystal.

\section{Bell-Operator Measurement and Teleportation}

The original BBCJPW teleportation procedure consists of three main stages: (i) Preparation of an EPR pair, (ii) Bell-operator measurement performed on the "input" particle and one particle of the EPR pair, (iii) Transmission of the outcome of the Bell measurement and appropriate unitary operation on the second particle of the EPR pair (the "output" particle). Completing (i)-(iii) ensures transportation of the pure state of the input particle to the output particle. It also ensures transportation of correlations: if the input particle were correlated to other systems, then the output particle ends up correlated to these systems in the same way.

The main difficulty in this procedure is performing the Bell measurement. Recently it has been proved [14, 15] that without "quantum-quantum" interaction one cannot perform 
measurement of the nondegenerate Bell operator which is required for reliable teleportation. Using only "quantum-classical" interactions one can perform a measurement of a degenerate Bell operator [16, 17], thus allowing a teleportation which succeeds sometimes.

The size limitations of this paper allow only to outline the proof [14. In order to prove that it is impossible to perform complete (nondegenerate) Bell-operator measurements without using interactions between quantum systems, I assume that any unitary transformation of single-particle states and any local single-particle measurement are allowed. There are four distinct (orthogonal) single-particle states involved in the definition of the Bell states: two channels, and a two-level system which enters into each channel. We name the channels left $(\mathrm{L})$ and right $(\mathrm{R})$, corresponding to the way the Bell states are written:

$$
\begin{aligned}
\left|\Psi_{ \pm}\right\rangle & =\frac{1}{\sqrt{2}}\left(|\uparrow\rangle_{L}|\downarrow\rangle_{R} \pm|\downarrow\rangle_{L}|\uparrow\rangle_{R}\right), \\
\left|\Phi_{ \pm}\right\rangle & =\frac{1}{\sqrt{2}}\left(|\uparrow\rangle_{L}|\uparrow\rangle_{R} \pm|\downarrow\rangle_{L}|\downarrow\rangle_{R}\right) .
\end{aligned}
$$

The measurement procedure can be divided into two stages: the unitary linear evolution, and local detection. The general form of the unitary linear evolution of the four singleparticle states can be written in the following manner:

$$
|\uparrow\rangle_{L} \rightarrow \sum a_{i}|i\rangle, \quad|\downarrow\rangle_{L} \rightarrow \sum b_{i}|i\rangle, \quad|\uparrow\rangle_{R} \rightarrow \sum c_{i}|i\rangle, \quad|\downarrow\rangle_{R} \rightarrow \sum d_{i}|i\rangle,
$$

where $\{|i\rangle\}$ is a set of orthogonal single-particle local states. The "linearity" implies that the evolution of the particle in one channel is independent on the state of the particle in another channel and, therefore, Eq. (2) is enough to define the evolution of the Bell states:

$$
\begin{aligned}
& \left|\Psi_{-}\right\rangle \rightarrow \sum_{i, j} \alpha_{i j}|i\rangle|j\rangle, \quad\left|\Psi_{+}\right\rangle \rightarrow \sum_{i, j} \beta_{i j}|i\rangle|j\rangle, \\
& \left|\Phi_{-}\right\rangle \rightarrow \sum_{i, j} \gamma_{i j}|i\rangle|j\rangle, \quad\left|\Phi_{+}\right\rangle \rightarrow \sum_{i, j} \delta_{i j}|i\rangle|j\rangle .
\end{aligned}
$$

Proper symmetrization is required for identical particles.

I assume that there are only local detectors and, therefore, only product states $|i\rangle|j\rangle$ (and not their superpositions) can be detected. Measurability of the non-degenerate Bell operator means that there is at least one nonzero coefficient of every kind $\alpha_{i j}, \beta_{i j}, \gamma_{i j}, \delta_{i j}$ and if, for a certain $i, j$, it is not zero, then all others are zero. This observation leads to numerous equations which, after some tedious algebra, yield the desired proof.

A somewhat different approach was taken in proof [15]. This proof considers only photons, but it proves the impossibility of non-degenerate Bell-measurements for a more general case in which measurements in two stages are allowed. The procedure in which the choice of the measurements in the second stage depends on the results of the measurements in the first stage is an indirect quantum-quantum interaction: the state of one quantum 
system influences the result of the first measurement and the action on the second quantum system depends on this result.

If we allow direct quantum-quantum interactions, we can achieve reliable (theoretically $100 \%$ efficient) teleportation. In this case, we can perform a measurement of the nondegenerate Bell operator. Indeed, a quantum-quantum interaction such as a conditional spin-flip transforms the Bell states into product states which then can be measured using single-particle measuring devices.

An alternative method of teleportation [18] is based on nonlocal measurements [19] "crossed" in space-time. In order to teleport a quantum state from particle 1 to particle 2 and, at the same time, the quantum state of particle 2 to particle 1, the following (nonlocal in space-time) variables should be measured (see Fig. 1):

$$
\mathcal{Z} \equiv\left(\sigma_{1 z}\left(t_{1}\right)+\sigma_{2 z}\left(t_{2}\right)\right) \bmod 4, \quad \mathcal{X} \equiv\left(\sigma_{1 x}\left(t_{2}\right)+\sigma_{2 x}\left(t_{1}\right)\right) \bmod 4
$$

For any set of outcomes of the nonlocal measurements (4) the spin state is teleported; in some cases the state is rotated by $\pi$ around one of the axes, but the resulting rotation can be inferred from the nonlocal measurements. In order to perform nonlocal measurements (4), correlated pairs of auxiliary particles located at the sites of particle 1 and 2 are required. For completing the whole procedure we need two singlets instead of the one required in the original teleportation procedure. The reason for requiring more resources is that two-way (rather than one-way) teleportation is achieved.

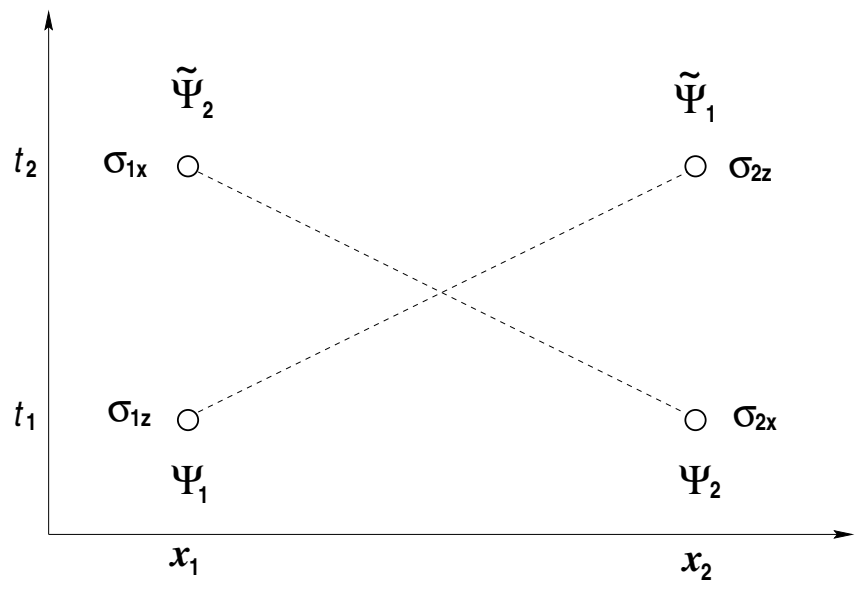

Fig. 1. Space-time diagram of "crossed" nonlocal measurements which result in two-way teleportation. Space-time locations of local couplings are shown. When the nonlocal measurements (4) are completed, the states of the two particles are interchanged up to local $\pi$ rotations, $\tilde{\Psi}_{i}$ signifies "rotated" $\Psi_{i}$. 


\section{Towards experimental realization of reliable tele- portation}

Due to the lack of an effective photon-photon interaction, the currently available methods do not allow reliable teleportation of the photon polarization state. It seems that the most promising candidates for teleportation experiments which might have $100 \%$ success rate are proposals which involve atoms and electro-magnetic cavities. First suggestions for such experiments [20, 21, 22] were made shortly after publication of the original teleportation paper and numerous modifications appeared since. The implementation of these proposals seems to be feasible because of the existence of the "quantum-quantum" interaction between the system carrying the quantum state and a system forming the EPR pair. A dispersive interaction (DI) of a Rydberg atom passing through a properly tuned micro-wave cavity leads to a conditional phase flip depending on the presence of a photon in the cavity. A resonant interaction (RI- $\pi$ ) between the Rydberg atom and the cavity allows swapping of quantum states of the atom and the cavity. Thus, manipulation of the quantum state of the cavity can be achieved via manipulation of the state of the Rydberg atom. The atom's state is transformed by sending it through an appropriately tuned microwave zone. Moreover, the direct analog of conditional spin-flip the interaction can be achieved through the Raman atom-cavity interaction [23]. No teleportation experiment has been performed as of yet using these methods, but it seems that the technology is not too far from this goal. Recent experiments on atom-cavity interactions [24] teach us about the progress in this direction.

Until further progress in technology is achieved, it is not easy to predict which proposal will be implemented first. Assuming that resonant atom-cavity interactions can be performed with very good precision and that a dispersive interaction is available with a reasonable precision, it seems that the following is the simplest proposal, see Fig. 2. The quantum channel consists of a cavity and a Rydberg atom in a correlated state. A particular resonant interaction, RI- $\pi / 2$, of an excited atom passing through an empty cavity,

$$
|e\rangle|0\rangle \rightarrow \frac{1}{\sqrt{2}}(|g\rangle|1\rangle+|e\rangle|0\rangle)
$$

prepares this quantum channel (Fig. 2a). The quantum state to be teleported is the state of another Rydberg atom. The Bell measurement is then performed on this atom and the cavity. To this end, the atom passes through the cavity interacting dispersively (Fig. $2 \mathrm{~b}$ ), induces the conditional phase flip,

$$
|e\rangle|0\rangle \rightarrow|e\rangle|0\rangle, \quad|e\rangle|1\rangle \rightarrow-|e\rangle|1\rangle, \quad|g\rangle|0\rangle \rightarrow|g\rangle|0\rangle, \quad|g\rangle|1\rangle \rightarrow|g\rangle|1\rangle,
$$

which disentangles the following Bell states:

$$
\begin{aligned}
&\left|\Psi_{ \pm}\right\rangle=\frac{1}{2}(|e\rangle(|0\rangle-|1\rangle) \pm|g\rangle(|1\rangle+|0\rangle)), \\
&\left|\Phi_{ \pm}\right\rangle=\frac{1}{2}(|e\rangle(|0\rangle+|1\rangle) \pm|g\rangle(|1\rangle-|0\rangle)) .
\end{aligned}
$$




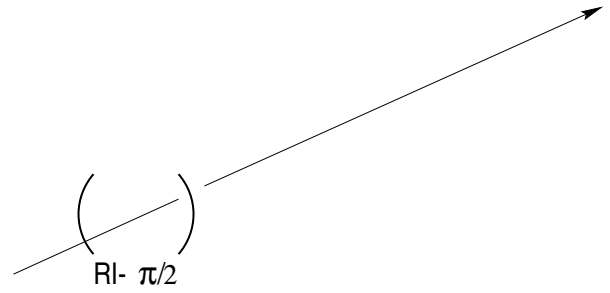

(a)

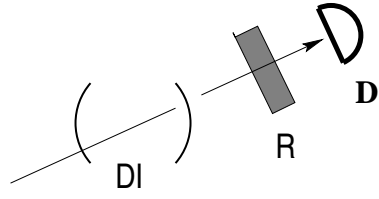

(b)

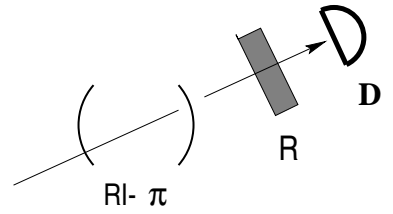

(c)

Fig. 2. Single-cavity teleportation of a quantum state of an atom.

(a) Preparation of the quantum channel. An atom undergoes resonant interaction RI- $\pi / 2$ with the cavity and moves to a remote site.

(b) The atom, carrying the quantum state to be teleported, interacts with the cavity dispersively and its state is measured.

(c) The state of the cavity is measured using an auxiliary atom.

The Bell states (7) have the form of Eq. 10 when the first $|\uparrow\rangle$ in the product is identified with $|e\rangle$, the second $|\uparrow\rangle$, with $(1 / \sqrt{2})(|0\rangle+|1\rangle)$, etc. Measurement of the atom state and the cavity state completes the Bell measurement procedure. In order to make the measurement of the cavity state we perform another resonant interaction, RI- $\pi$, between the cavity and an auxiliary atom prepared initially in the ground state (Fig. 2c),

$$
|g\rangle|1\rangle \rightarrow|e\rangle|0\rangle, \quad|g\rangle|0\rangle \rightarrow|g\rangle|0\rangle .
$$

This interaction transfers the quantum state of the cavity to this atom. The final measurements on the atoms distinguish between the states $(1 / \sqrt{2})(|g\rangle+|e\rangle)$ and $(1 / \sqrt{2})(|g\rangle-|e\rangle)$. Since detectors can distinguish between $|g\rangle$ and $|e\rangle$, the states of the atoms are rotated while passing through the appropriate microwave zones before detection. When the Bell measurement is completed, the quantum state is teleported up to the known local transformation determined by the results of the Bell measurement. (This final local transformation is not shown in Fig. 2.)

One relatively simple method for "two-way" teleportation of atomic states is a direct implementation of the crossed nonlocal measurement scheme presented in the previous section. This method is described in Ref. [14].

One difficulty with the teleportation of atomic states is that usually experiments are performed with atomic beams and not with individual atoms. Such experiments might be good for demonstration and studying experimental difficulties of teleportation, but they cannot be considered as implementation of the original wisdom of teleportation or used for cryptographic purposes. In fact, optical experiments have this difficulty too, unless "single-photon guns" will be used. Both for atomic and for optical experiments this difficulty does not seem to be unsolvable, but it certainly brings attention to experiments with trapped ions [25]. There are many similarities between available manipulations with atoms and with ions, so the methods discussed above might be implemented for ion systems too. 
Note also another recent proposal for teleportation using quantum-quantum interaction [26]. It is based on rotation of the photon polarization due to presence of a single chiral molecule in an optical cavity. I am, however, skeptical about the feasibility of such experiment due to difficulties in tuning the interferometer in which photons undergo multiple reflections in the cavity; the number of reflections has to be very large due to weakness of the interaction between the molecule and the photon.

\section{Teleportation of continuous variables}

In the framework of nonlocal measurements there is a natural way of extending the teleportation scheme to systems with continuous variables [18]. Consider two similar systems located far away from each other and described by continuous variables $q_{1}$ and $q_{2}$ with corresponding conjugate momenta $p_{1}$ and $p_{2}$. In order to teleport the quantum state of the first particle $\Psi_{1}\left(q_{1}\right)$ to the second particle (and the state of the second particle $\Psi_{2}\left(q_{2}\right)$ to the first) we perform the following "crossed" nonlocal measurements (see Fig. $3)$, obtaining the outcomes $a$ and $b$ :

$$
q_{1}\left(t_{1}\right)-q_{2}\left(t_{2}\right)=a, \quad p_{1}\left(t_{2}\right)-p_{2}\left(t_{1}\right)=b .
$$

In Ref. 18] it is shown that these nonlocal "crossed" measurements "swap" the quantum states of the two particles up to the known shifts in $q$ and $p$. Indeed, the states of the particles after completion of the measurements (9) are

$$
\Psi_{f}\left(q_{1}\right)=e^{i b q_{1}} \Psi_{2}\left(q_{1}+a\right), \quad \Psi_{f}\left(q_{2}\right)=e^{-i b q_{2}} \Psi_{1}\left(q_{2}-a\right) .
$$

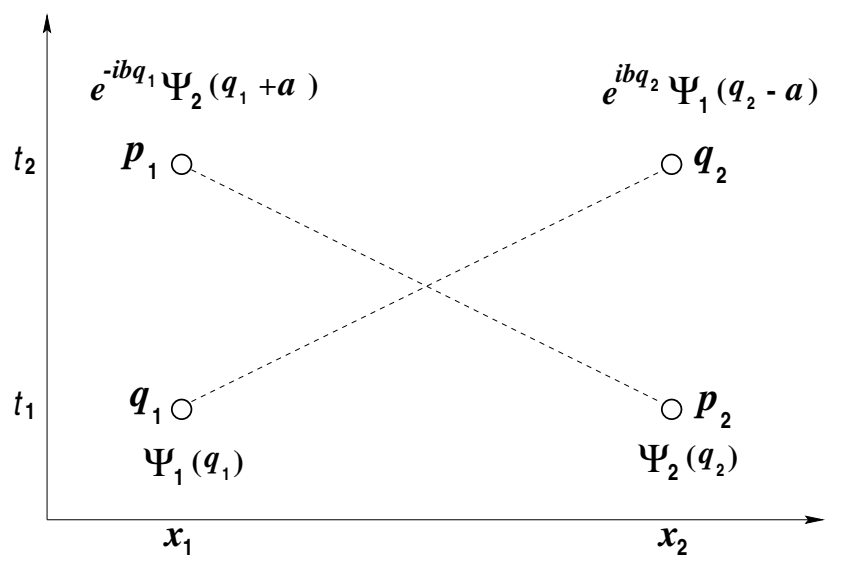

Fig. 3. Space-time diagram of "crossed" nonlocal measurements which result in two-way teleportation of quantum states of quantum systems with continuous variables. Space-time locations of local couplings are shown. When the nonlocal measurements (9) are completed, the states of the two particles are interchanged up to the known shifts in $q$ and $p$. 
The state of particle 2 after $t_{2}$ is the initial state of the particle 1 shifted by $-a$ in $q$ and by $-b$ in $p$. Similarly, the state of particle 1 is the initial state of particle 2 shifted by $a$ in $q$ and by $b$ in $p$. After transmitting the results of the local measurements, $a$ and $b$, the shifts can be corrected (even if the quantum state is unknown) by appropriate kicks and back shifts, thus completing a reliable teleportation of the state $\Psi_{1}\left(q_{1}\right)$ to $\Psi_{1}\left(q_{2}\right)$ and of the state $\Psi_{2}\left(q_{2}\right)$ to $\Psi_{2}\left(q_{1}\right)$.

Surprisingly, the implementation of the reliable teleportation of continuous variables is possible. Braunstein and Kimble made a realistic proposal for teleporting the quantum state of a single mode of the electro-magnetic field [27]. This remarkable result is an implementation of a variation of the scheme described above which achieves a one-way teleportation. In their method $q$ is " $x$ " defined for a single mode of an electro-magnetic field, and correspondingly $p$ is the conjugate momentum of $x$. The analog of the EPR pair is obtained by shining squeezed light with a certain $x$ from one side and squeezed light with a certain $p$ from the other side onto a simple beam splitter. The analog of the local Bell measurement is achieved using another beam splitter and homodyne detectors. The shifts in $x$ and $p$ which complete the teleportation procedure can be done by combining the output field with the coherent state of appropriate amplitude fixed by the results of the homodyne measurements. Note also a related proposal [29, 30] for teleporting a single-photon wave packet.

Very recently the Braunstein-Kimble proposal for implementation of continues variable teleportation [18 has been performed in California Institute of Technology [28]. This is the first reliable teleportation experiment. The meaning of "reliable" ("unconditional" in [28]) is that theoretically it is always successful. It is the first experiment in which the final stage of teleportation, i.e., transmission of the classical information to Bob and the appropriate transformation which results in the appearance of the teleported state in the Bob's site, has been implemented. The weakness of this experiment is that the teleported state is significantly distorted. The main reason for low fidelity is the degree of squeezing of the light which controls the quality of the EPR pairs, the quantum channel of the teleportation. Significant improvement of the squeezing parameters is a very difficult technological problem. Thus, in this type of experiment one cannot reach the high fidelity of (conditional) teleportation experiments of photon polarization states.

One may note an apparent contradiction between the proof of Section 3 that $100 \%$ efficient teleportation cannot be achieved using linear elements and single-particle state detectors and the successful reliable teleportation experiment of the state of the electromagnetic field which involved only beam-splitters and local measuring devices reported above. Indeed, it is natural to assume that if reliable teleportation of a quantum state of a two-level system is impossible under certain circumstances, it should certainly be impossible for quantum states of systems with continuous variables. However, although it is not immediately obvious, the circumstances are very different. There are numerous differences. The analog of the Bell operator for continuous variables does not have among its eigenvalues four states of the general form (1) where $|\uparrow\rangle$ and $|\downarrow\rangle$ signify some orthogonal 
states. Another problem is that one cannot identify "the particles": In the beam-splitter one input port goes to two output ports. One can see a "quantum-quantum" interaction: the variable $x$ of one of the output ports of the beam splitter becomes equal to $(1 / \sqrt{2})\left(x_{1}+\right.$ $\left.x_{2}\right)$, essentially, the sum of the quantum variables of the input ports. The absence of such "quantum-quantum" interactions is an essential ingredient in the proof of Section 3. If, however, we consider the "particles" to be photons (which do not interact with one another) then the homodyne detectors which measure $x$ are not single-particle detectorsanother constraint used in the proof. Note also that the Braunstein-Kimble method is not applicable directly for teleporting $\Psi(x)$ where $x$ is a spatial position of a quantum system. An additional quantum-quantum interaction which converts the continuous variable of position of a particle to the variable $x$ of the electro-magnetic mode is required.

\section{Is There a Paradox with Teleportation?}

My complaints about the (mis)interpretation of the word "teleportation" in Section II shows that I am (over)sensitive about this issue. This is because I was thinking a lot about it, resolving for myself a paradox [31] which I, as a believer in the Many-Worlds Interpretation (MWI) 32] had with this experiment.

Consider teleportation, say in the BBCJPW scheme. We perform some action in one place and the state is immediately teleported, up a local transformation ("rotation"), to an arbitrary distant location. But relativity theory teaches us that anything which is physically significant cannot move faster than light. Thus it seems that it is the classical information (which cannot be transmitted with superluminal velocity) about the kind of back "rotation" to be performed for completing the teleportation which is the only essential part of the quantum state. However, the amount of the required classical information is very small. Is the essence of a state of a spin- $1 / 2$ particle just 2 bits?

I tend to attach a lot of physical meaning to a quantum state. For me, a proponent of the MWI, everything is a quantum state. But I also believe in relativistic invariance, so only entities which cannot move faster than light have physical reality. Thus, teleportation poses a serious problem to my attitude. I was ready to admit that "I" am just a quantum state of $N \sim 10^{30}$ particles. This is still a very rich structure: a complex function on $\mathcal{R}^{N}$. But now I am forced to believe that "I" am just a point in the $\mathcal{R}^{2 N}$ ?!

The resolution which I found for myself is as follows: In the framework of the MWI, the teleportation procedure does not move the quantum state: the state was, in some sense, in the remote location from the beginning. The correlated pair, which is the necessary item for teleportation, incorporates all possible quantum states of the remote particle, and, in particular, the state $\Psi$ which has to be teleported. The local measurement of the teleportation procedure splits the world in such a manner that in each of the worlds the state of the remote particle differs form the state $\Psi$ by some known transformation. The number of such worlds is relatively small. This explains why the information which has to be transmitted for teleportation of a quantum state - the information which world we 
need to split into, i.e., what transformation has to be applied - is much smaller than the information which is needed for the creation of such a state. For example, for the case of a spin-1/2 particle there are only 4 different worlds, so in order to teleport the state we have to transmit just 2 bits. As for teleporting myself, the number of worlds is the number of distinguishable (using measuring devices and our senses) values of $x_{i}$ and $p_{i}$ for all continues degrees of freedom of my body.

Teleportation of people will remain a dream for the foreseeable future. First, we have to achieve the reliable teleportation of an unknown quantum state of an external system with reasonable fidelity which is also only a dream today. Although the teleportation of an unknown quantum state has not yet been achieved, the current experiments clearly demonstrate that it can be done. I urge the experimenters to perform a persuasive teleportation experiment: Carol gives to Alice (single) particles in different states (unknown to Alice), Alice teleports the states to Bob, Bob gives them back to Carol who tests that what she gets is what she has sent before.

I am grateful for very useful correspondence with Chris Adami, Gilles Brassard, Samuel Braunstein, John Calsamiglia, Lior Goldenberg, Daniel Lidar, Sergey Molotkov, Harald Weinfurter, Asher Peres, Sandu Popescu, and Anton Zeilinger. The research was supported in part by grant 471/98 of the Basic Research Foundation (administered by the Israel Academy of Sciences and Humanities).

\section{References}

[1] The Oxford English Dictionary, 2nd Ed. (Clarendon Press, Oxford 1989) XVII p. 730 .

[2] C. H. Bennett, G. Brassard, C. Crepeau, R. Jozsa, A. Peres, W. K. Wootters, Phys. Rev. Lett. 70, 1895 (1993).

[3] R. Laflamme, Talk given at the Benasque Workshop on Quantum Computation, July 1998.

[4] N. J. Cerf, C. Adami, P. G. Kwiat, Phys. Rev. A 57, R1477 (1998).

[5] G. Brassard, e-print quant-ph/9605035 (1996).

[6] G. Brassard, S. L. Braunstein and R. Cleve, Physica D 120, 43 (1998).

[7] D. Boschi, S. Branca, F. De Martini, L. Hardy and S. Popescu, Phys. Rev. Lett. 80, 1121 (1997).

[8] S. Popescu, e-print quant-ph/9501020.

[9] D. Bouwmeester, J. W. Pan, K. Mattle, M. Eibl, H. Weinfurter, A. Zeilinger, Nature 390, 575 (1997).

[10] J. W. Pan, D. Bouwmeester, H. Weinfurter, and A. Zeilinger Phys. Rev. Lett. 80, 3891 (1998). 
[11] S. L. Braunstein, H. J. Kimble, Nature 394, 840 (1998).

[12] D. Bouwmeester, J. W. Pan, K. Mattle, M. Eibl, H. Weinfurter, A. Zeilinger, Nature 394, 840 (1998).

[13] L. Vaidman, in The Geometric Universe, S. Huggett et al. eds., Oxford University Press, Oxford, p. 349 (1998).

[14] L. Vaidman and N. Yoran, Phys. Rev. A, to be published, quant-ph/9808043.

[15] N. Lütkenhaus, J. Calsamiglia, K-A. Suominen, e-print quant-ph/9809063.

[16] H. Weinfurter, Europhys. Lett. 559 (1994).

[17] S. L. Braunstein and A. Mann, Phys. Rev. A 51, R1727 (1955).

[18] L. Vaidman, Phys. Rev. A 49, 1473 (1994).

[19] Y. Aharonov, D. Albert, and L.Vaidman, Phys. Rev. D 34, 1805 (1986).

[20] T. Sleator and H. Weinfurter, in IQEC Technical Digest 1994, Vol. 9, 1994, OSA Technical Digest Series (OSA, Washington, D.C., 1994), p. 140.

[21] L. Davidovich, N. Zagury, M. Brune, J. M. Raimond and S. Haroche, Phys. Rev. A, 50, 895, (1994).

[22] J. I. Cirac and A. S. Parkins, Phys. Rev. A 50 R4441 (1994).

[23] S. B. Zheng, G. C. Guo, Phys. Lett. A 232, 171 (1997).

[24] S. Haroche, M. Brune, J. R. Raimond, Philos. T. Roy. Soc. A 355 (1733) 2367 (1997).

[25] R. J. Hughes, Philos. T. Roy. Soc. A 356 (1743) 1853 (1998).

[26] C. S. Maierle, D. A. Lidar, R. A. Harris, e-print quant-ph/9807020.

[27] S. L. Braunstein, H. J. Kimble, Phys. Rev. Lett. 80, 869 (1998).

[28] A. Furusawa, J. Sørenson, S. L. Braunstein, C. A. Fuchs, H. J. Kimble and E. S. Polzik, Science, 282, 706 (1998).

[29] S. N. Molotkov, Phys. Lett. A 245, 339 (1998).

[30] S. N. Molotkov, JETP Lett. 68, 263 (1998).

[31] L. Vaidman, in PSA 1994, D. Hull, M. Forbes and R. M. Burian eds., (East Lansing, MI 1994) vol. 1, p. 211.

[32] L. Vaidman, Int. Stud. Phil. Sci. 12, (1998). 